\title{
Effect of dietary water intake on urinary output, specific gravity and relative supersaturation for calcium oxalate and struvite in the cat
}

\author{
Catherine M. F. Buckley, Amanda Hawthorne, Alison Colyer and Abigail E. Stevenson* \\ Waltham Centre for Pet Nutrition, Freeby Lane, Waltham-on-the-Wolds, Melton Mowbray, Leicestershire LE14 4RT, UK
}

(Received 13 October 2010 - Revised 27 January 2011 - Accepted 13 February 2011)

\begin{abstract}
It has been reported that daily fluid intake influences urinary dilution, and consequently the risk of urolithiasis in human subjects and dogs. The aim of the present study was to investigate the role of dietary moisture on urinary parameters in healthy adult cats by comparing nutritionally standardised diets, varying only in moisture content. A total of six cats were fed a complete dry food (6.3\% moisture) hydrated to $25.4,53 \cdot 2$ and $73.3 \%$ moisture for 3 weeks in a randomised block cross-over design. Urinary specific gravity (SG), urine volume, water drunk and total fluid intake were measured daily; relative supersaturation (RSS) for calcium oxalate (CaOx) and struvite was calculated using the SUPERSAT computer program. Cats fed the $73.3 \%$ moisture diet produced urine with a significantly lower SG $(P<0 \cdot 001)$ compared with diets containing $53 \cdot 2 \%$ moisture or lower. Mean RSS for CaOx was approaching the undersaturated zone (1·14 (sEM $0 \cdot 21$ ); $P=0.001$ ) for cats fed the diet with $73 \cdot 3 \%$ moisture and significantly lower than the $6 \cdot 3 \%$ moisture diet (CaOx RSS $2 \cdot 29$ (SEM $0 \cdot 21$ )) The effect of diet on struvite RSS was less clear, with no significant difference between treatment groups. Total fluid intake was significantly increased $(P<0.001)$ in the $73.3 \%$ moisture diet $(144.7$ (SEM 5.2$) \mathrm{ml}$, or $30 \mathrm{ml} / \mathrm{kg}$ body weight per $\mathrm{d})$ compared with the $6 \cdot 3 \%(103.4$ $(\operatorname{sem} 5.3) \mathrm{ml}), 25.4 \%(98.6(\operatorname{sem} 5.3) \mathrm{ml})$ and $53.3 \%(104.7(\operatorname{sem} 5.3) \mathrm{ml})$ moisture diets, despite voluntary water intake decreasing as dietary moisture intake increased. Cats fed the $73.3 \%$ moisture diet had a higher total daily fluid intake resulting in a more dilute urine with a lower risk of $\mathrm{CaOx}$ when compared with the lower-moisture diets.
\end{abstract}

The extent to which cats are able to adjust the amount of water they drink in relation to the moisture content of their diet has been a source of investigation over the last 30 years. Cats fed an all meat or wet diet, which typically contains in excess of $75 \%$ moisture, will voluntarily drink only small quantities of water ${ }^{(1,2)}$. Although cats drink more water when offered dry diets, they do not drink amounts that would be expected to compensate for the significant reduction in moisture content of these diets ${ }^{(2)}$.

It is believed that cats adjust their water intake such that they effectively excrete their renal solute load. Since cats fed dry food have a lower total water intake than when fed wet food, it would suggest that the absolute water requirement to dilute the renal solute load is lower than that resulting from a wet-food diet. This means that cats fed wet or natural diets may consume water in excess of any physiological requirement.

Dry cat foods have been implicated as a risk factor for cats susceptible to feline lower urinary tract disease ${ }^{(3)}$, while feeding wet diets has been shown to reduce the recurrence of calculi and signs of idiopathic cystitis compared with dry food ${ }^{(4)}$.
While the benefits of wet food are thought to be attributed to the increased water intake and related increase in urine volume, additional nutritional differences exist between wet and dry diets that may also be responsible for some of these benefits. Only a single study has previously been conducted to investigate solely the effect of dietary moisture in cats, although this used a semi-purified diet ${ }^{(5)}$.

The aim of the present study was to investigate the role of dietary moisture on urine parameters and total daily fluid intake in healthy adult cats by comparing nutritionally standardised diets, varying only in moisture content.

\section{Materials and methods}

A panel of six healthy neutered adult cats was studied, consisting of three males and three females, ages ranging from 2 years and 7 months to 6 years and 9 months. The experimental procedures were approved by the WALTHAM ${ }^{\circledR}$ (Melton Mowbray, Leicestershire, UK) ethical review committee. A single batch of complete and balanced dry diet was used in the present study $(6.3 \%$ moisture $)$ and this was soaked with de-ionised water where necessary to achieve dietary moisture

Abbreviations: CaOx, calcium oxalate; RSS, relative supersaturation; SG, specific gravity.

*Corresponding author: A. E. Stevenson, fax +44 1664 415440, email abigail.stevenson@effem.com 
contents of $25 \cdot 4,53 \cdot 2$ and $73 \cdot 3 \%$, with cats allocated to each of the four diets in a randomised block design. The cats were offered $188.3 \mathrm{~kJ} / \mathrm{kg}$ ( $45 \mathrm{kcal} / \mathrm{kg}$ ) body weight in each of the four phases split into three meals over the course of the day. Each trial phase lasted for 3 weeks during which food intake, water intake (water drunk plus dietary water), faeces quality, urine volume and specific gravity (SG) were recorded daily and urinary $\mathrm{pH}$ was measured using a $24 \mathrm{~h}$ monitoring system. The first $7 \mathrm{~d}$ data from each trial phase were discarded from any analysis, allowing for a period of adaptation. A $48 \mathrm{~h}$ urine sample was collected on days 17-19 in each phase. The urine sample was frozen immediately upon collection using dry ice and titrated down to $\mathrm{pH} 2$ with concentrated $\mathrm{HCl}$. The sample was then analysed for $\mathrm{Na}, \mathrm{K}, \mathrm{Mg}, \mathrm{Ca}, \mathrm{Cl}$, sulphate, phosphate, oxalate, citrate, pyrophosphate, $\mathrm{NH}_{3}$ and urate. These data were analysed using the computer-based program SUPERSAT $^{(6)}$ to calculate relative supersaturation (RSS) of the urine for calcium oxalate $(\mathrm{CaOx})$ and struvite.

Mean trial data for each cat were used in all analyses rather than individual data points. Error is expressed as standard errors of the mean. For the RSS measurements, ANOVA was performed using cat and diet as main effects. For SG, urine volume, water drunk and total water intake, a mixed model analysis was performed to allow for the repeated measures on each cat, within a diet. Thus, diet in cat was added as a random effect. The day $\times$ diet interaction was added as a fixed effect to investigate whether any effect of diet changed from day 8 to 21. Adjustments for the water drunk, as a covariate, were investigated but dropped from the model as appropriate. To allow for a six pairwise comparison of the data, a Bonferroni correction was made and is included where it alters the data interpretation. Statistical analyses were performed using Statgraphics Centurion XVI and GenStat ${ }^{\circledR}$ version 12.2 statistical software.

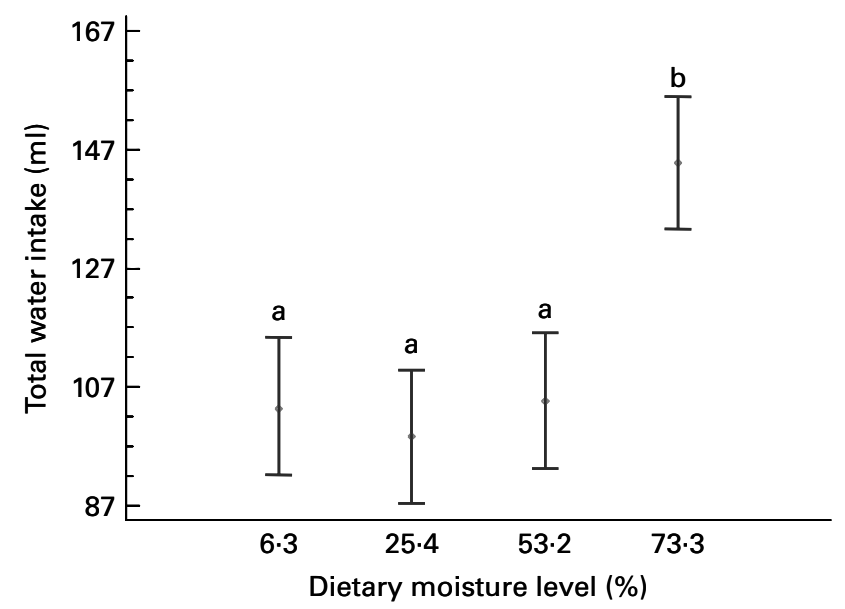

Fig. 1. Effect of dietary moisture on total water intake. Values are means, with $95 \% \mathrm{Cl}$ represented by vertical bars. ${ }^{\mathrm{a}, \mathrm{b}}$ Mean values with unlike letters were significantly different $(n 6)$.

\section{Results}

The effect of phase was found to be non-significant and was subsequently dropped from the model. The interaction effect of day $\times$ diet was not significant overall for SG, water drunk, urine volume and water intake and was therefore dropped from the model. Over the course of the present study, the cats showed a mean increase in body weight of $8 \cdot 03+3 \cdot 16 \%$, despite being offered only $188.3 \mathrm{~kJ} / \mathrm{kg}$ per $\mathrm{d}$ ( $45 \mathrm{kcal} / \mathrm{kg}$ per d). Faeces quality was excellent, with $100 \%$ of faeces voided being acceptable across each phase.

As dietary moisture level increased, the cats compensated by significantly reducing the amount of water they drunk voluntarily. There was a significant increase in total water intake $(P<0.001)$ for the diets with $73.3 \%$ moisture compared with all other diets. For the highest moisture diet, mean total water intake was 144.7 (SEM $5 \cdot 2$ ) $\mathrm{ml}$ or $30 \mathrm{ml} / \mathrm{kg}$ body weight per d compared with $103 \cdot 4$ (SEM 5.3), $98 \cdot 6$ (SEM 5.3) and 104.7 (sem 5.3) $\mathrm{ml}$ for the 6.3, 25.4 and $53.3 \%$ moisture diets, respectively (Fig. 1).

The average $24 \mathrm{~h}$ urine volume produced by cats fed the diet containing $73.3 \%$ moisture was 86.7 (SEM 6.93) $\mathrm{ml}$, which was significantly higher than all other diets $(P<0 \cdot 001)$. Average urine volume did not differ significantly for any of the other diets $(P>0.05)$.

Mean $48 \mathrm{~h}$ urinary $\mathrm{pH}$ showed no statistically significant differences according to the diet group (6.22 (SEM 0.053); $6 \cdot 23$ (SEM 0.034); 6.24 (SEM 0.061) and 6.19 (SEM 0.042), respectively for the treatment groups as described previously). For SG, the day $\times$ diet interaction and the main effect of day were found to be non-significant and were dropped from the model. Following this, the effect of diet was interpreted as significant $(P<0.001)$. There was a significant reduction in mean SG for the $73.3 \%$ moisture diet (1.036 (SEM 0.002)) compared with all other diets (1.052-1.054 (SEM 0.002); $P<0.001)$. All other diets were not significantly different $(P>0.05)$.

The mean CaOx RSS for the $73.3 \%$ moisture diet was significantly reduced $(1 \cdot 14(\operatorname{sem} 0 \cdot 21))$ compared with the $6 \cdot 3 \%(2 \cdot 29$ (SEM $0 \cdot 21)$ ) and $53.2 \%$ moisture diets (2.06 (SEM 0.21)). The 73 v. $25 \cdot 4 \%$ diet was non-significant, following a Bonferroni correction.

The effect of diet on struvite RSS was not significant overall $(P=0 \cdot 155)$. By performing pairwise comparisons, the average RSS struvite for the $73.3 \%$ diet was significantly lower than the 53.2 and $6.3 \%$ diets $(P=0.045$ and 0.035 , respectively). All other diets were not significantly different $(P>0.05)$. When a Bonferroni correction to the data was made, the test level became $0 \cdot 008$, making none of the diets significantly different.

\section{Discussion}

The key findings from the present study were that high dietary water intake is related to a significant increase in urine volume, reduction in SG and decrease in CaOx RSS, demonstrating a beneficial effect of high-moisture diets on cat urinary parameters. 
The data from the present study show that water intake alone has a strong influence on urine parameters. This is in agreement with Gaskell $^{(5)}$ who demonstrated that addition of water to a semi-purified diet to achieve a moisture level of $75 \%$ produced a similar water intake and urine SG to the present study. Increasing water intake has been identified as an important strategy in the management of patients with urolithiasis, primarily because of its role in increasing urine volume. The effect of this is a decrease in saturation through dilution of calculogenic material ${ }^{(7)}$, and an increase in the minimum supersaturation required to elicit initiation of crystallisation $^{(8,9)}$, and these benefits outweigh the potential negative effects of dilution of inhibitors of crystallisation or growth ${ }^{(10)}$. In the present study, each dietary moisture level would have offered the same calculogenic load, because the cats were offered the same amount of food differing only in the levels of water added. This suggests that any effects observed on urine can be attributed to varying degrees of urine concentration as dietary moisture levels are altered, rather than any nutritional differences.

When considering the natural behaviour of the cat's ancestor, much of their daily water requirement is obtained from their prey (which typically contains $70-75 \%$ moisture) and cats have evolved to drink very little water. As a result, cats naturally have a very low thirst drive and are therefore slow to respond to changes in their hydration state. This reluctance to adapt their voluntary water intake sufficiently is illustrated in the present study where cats fed the dry diet (6.3\% moisture) consumed approximately $30 \%$ less water overall compared with cats fed on the highest-moisture diet, despite the dry-diet group increasing their voluntary drinking by approximately sixfold in comparison. This behaviour puts cats at a higher risk of lower urinary tract disease when fed low-moisture diets, based on a more concentrated urine and an associated increase in $\mathrm{CaOx}$ RSS. Since energy intake remained constant across all four diets, these data suggest that the high-moisture (and less energy-dense) diet may drive cats to ingest more dietary moisture than is required in order to meet their daily energy requirements with the consequence of positive effects on urinary parameters.

The significant reduction in CaOx RSS in cats fed the highest-moisture diet demonstrates that increased water turnover is of real benefit in cats. In terms of the biological relevance of the observations made in the present study, the high-moisture diet resulted in the production of urine approaching the undersaturated zone for CaOx RSS, which is defined as $<1$, reducing the risk of both homogeneous and heterogeneous $\mathrm{CaOx}$ crystallisation ${ }^{(11)}$. The other diets resulted in mean CaOx RSS values ranging from 1.99 to $2 \cdot 29$, classified as within the metastable zone (RSS $=1-12$ ), indicating that new $\mathrm{CaOx}$ stone formation in these cats is possible if fed these diets in the long term. The present study was underpowered, and it is expected that statistically significant differences in struvite RSS would be found in the highest-moisture diet in a suitably powered study. Since literary evidence shows that urinary $\mathrm{pH}$ correlates with struvite $\mathrm{RSS}^{(12)}$, the lack of significant differences in urinary $\mathrm{pH}$ across dietary groups in the present study may explain the similar findings for struvite RSS and indicates that urinary $\mathrm{pH}$ may be a more important driver of struvite RSS than SG.

\section{Acknowledgements}

There are no conflicts of interest to disclose. The study received no specific grant from any funding agency in the public, commercial or not-for-profit sectors. C. M. F. B. prepared the manuscript. A. C. prepared the statistical analyses. A. E. S. and A. H. conducted the study.

\section{References}

1. Thrall BE \& Miller LG (1976) Water turnover in cats fed dry rations. Fel Pract 6, 10-17.

2. Burger IH, Anderson RS \& Holme DW (1980) Nutritional factors affecting water balance in the dog and cat. In Nutrition of the Dog and Cat, pp. 145-156 [RS Anderson, editor]. Oxford: Pergamon Press.

3. Buffington CAT, Chew DJ, Kendall MS, et al. (1997) Clinical evaluation of cats with non-obstructive urinary tract diseases. J Am Vet Med Assoc 210, 46-50.

4. Markwell PJ, Buffington CAT, Chew DJ, et al. (1999) Clinical evaluation of commercially available urinary acidification diets in the management of idiopathic cystitis in cats. $J \mathrm{Am}$ Vet Med Assoc 214, 361-365.

5. Gaskell CJ (1985) Nutrition in diseases of the urinary tract in the dog and cat. Vet Annual 25, 383-390.

6. Robertson WG, Jones JS, Heaton MA, et al. (2002) Predicting the crystallisation potential of urine from cats and dogs with respect to calcium oxalate and magnesium ammonium phosphate (struvite). J Nutr 132, 1637S-1641S.

7. Borghi L, Meschi T, Amato F, et al. (1996) Urinary volume, water and recurrences in idiopathic calcium nephrolithiasis: a 5-year randomised prospective study. J Urol 155, 839-843.

8. Sakhaee K, Zerweke JE, Pak CYC, et al. (1981) Objective evidence for the beneficial effect of a high fluid intake in the management of nephrolithiasis. In Urolithiasis: Clinical and Basic Research, pp. 227-233 [LH Smith, WG Robertson and B Finlayson, editors]. New York: Plenum Press.

9. Pak CYC, Sakhaee K, Crowther C, et al. (1980) Evidence justifying a high fluid intake in treatment of nephrolithiasis. Ann Int Med 93, 36-39.

10. Borghi L, Meschi T, Schianchi T, et al. (1999) Urine volume: stone risk factor and preventive measure. Nephron $\mathbf{8}$, 131-137.

11. Hurley K, Stevenson A \& Watson H (2003) Managing struvite and calcium oxalate risk - what does relative super saturation (RSS) evaluation mean in practical terms? Waltham Focus 13, 30-33.

12. Osborne CA, Kruger JM, Lulich JP, et al. (2000) Feline lower urinary tract diseases. In Textbook of Veterinary Internal Medicine, pp. 1710-1746 [S Ettinger and E Feldman, editors]. Philadelphia, PA: WB Saunders. 